Article

\title{
Increased Omega-3 Fatty Acid Intake is Inversely Associated with Sarcopenic Obesity in Women but not in Men, Based on the 2014-2018 Korean National Health and Nutrition Examination Survey
}

\author{
Woojung Yang ${ }^{1}{ }^{\oplus}$, Jae-woo Lee ${ }^{1} \oplus$, Yonghwan Kim ${ }^{1} \oplus$, Jong Hun Lee ${ }^{2} \oplus$ and \\ Hee-Taik Kang 1,3,*(D) \\ 1 Department of Family Medicine, Chungbuk National University Hospital, Cheongju 28644, Korea; \\ kineto@naver.com (W.Y.); shrimp0611@gmail.com (J.-w.L.); airsantajin@gmail.com (Y.K.) \\ 2 Department of Food Science and Biotechnology, Gachon University, Seongnam 13120, Korea; \\ foodguy@gachon.ac.kr \\ 3 Department of Family Medicine, Chungbuk National University College of Medicine, Cheongju 28644, Korea \\ * Correspondence: kanght0818@gmail.com; Tel.: +82-43-269-6301
}

Received: 21 October 2020; Accepted: 25 November 2020; Published: 27 November 2020

\begin{abstract}
Background: Omega-3 fatty acids (w3FAs) are known to improve protein anabolism, increase the sensitivity to anabolic stimuli, decrease lipogenesis, and stimulate lipid oxidation. We aim to investigate whether w3FAs are associated with the prevalence of sarcopenic obesity (SO). (2) Methods: Data were obtained from the 2014-2018 Korean National Health and Nutrition Examination Survey. The ratio of daily $\omega 3 \mathrm{FA}$ intake to energy intake ( $\omega 3 \mathrm{FA}$ ratio) was categorized into four quartile groups. (3) Results: The prevalence of SO from Q1 to Q4 was 8.9\%, 11.3\%, $11.0 \%$, and $9.8 \%$ respectively, in men and $17.4 \%, 14.0 \%, 13.9 \%$, and $10.1 \%$ respectively, in women. The $\omega 3$ FA ratio in individuals with and without SO were $1.0 \%$ and $0.9 \%$ in men $(p$-value $=0.271)$ respectively, and $0.8 \%$ and $1.0 \%$ in women ( $p$-value $=0.017)$, respectively. Compared with Q1, odds ratios $(95 \%$ confidence intervals) of Q2, Q3, and Q4 of $\omega 3$ FA ratios were 1.563 (0.802-3.047), 1.246 (0.611-2.542), and $0.924(0.458-1.864)$ respectively, in men and $0.663(0.379-1.160), 0.640(0.372-1.102)$, and 0.246 (0.113-0.534) respectively, in women, after fully adjusting for confounding factors. (4) Conclusions: The $\omega 3$ FA ratio was significantly higher in older females without SO than in older females with SO. The $\omega 3$ FA ratio was associated with the prevalence of $\mathrm{SO}$ in elderly females.
\end{abstract}

Keywords: omega-3 fatty acids; sarcopenic obesity; omega-3 fatty acid ratio; sarcopenia

\section{Introduction}

The greatest epidemiological trend in Korea in the 21st century is the unprecedented growth of the rapidly aging population. In general, aging leads to a progressive decrease in muscle mass (sarcopenia) and an increase in fat mass (obesity) [1]. Korea, with the most rapidly aging population in the world, may confront a massive increase in the prevalence of sarcopenic obesity (SO). Sarcopenia and obesity in the elderly are frequently related to physical disability and visceral fat accumulation, displaying a synergistic interaction that can lead to a vicious cycle [2,3]. As a result, concurrent sarcopenia and obesity in elderly people increase all-cause mortality and lead to worse health outcomes than sarcopenia or obesity alone [4].

Omega-3 fatty acids ( $\omega 3 \mathrm{FAs}$ ) are known to improve net muscle protein anabolism by activating the mammalian target of rapamycin/ribosomal protein kinase S6 (mTORp/70s6k) signaling pathway $[5,6]$. This signaling pathway increases the sensitivity of responses to anabolic stimuli such as enhanced 
protein intake, resistance exercise, and insulin [5,6]. On the other hand, $\omega 3$ FAs are also found to downregulate lipogenesis by inhibiting differentiation of adipocytes by competing with prostacyclin (PGI2) in downstream $[7,8]$ and to stimulate basal lipid oxidation by increasing the activity of peroxisomal acyl-CoA oxidase $[9,10]$.

In some recent studies, dietary supplementation with $\omega 3$ FAs has been shown to significantly decrease muscle mass loss and obesity $[5,6]$. Furthermore, it is noteworthy that adequate protein intake above the recommended daily intake (RDI) alone cannot guarantee prevention of sarcopenia [11-13]. These previous studies, however, have some limitations in that they have been implemented only in animals and in small sample sizes of people.

Therefore, the purpose of this study is to evaluate whether the ratio of daily $\omega 3$ FA consumption to daily energy intake is associated with the prevalence of $\mathrm{SO}$ in elderly people in Korea based on the 2014-2018 Korean National Health and Nutrition Examination Survey (KNHANES).

\section{Materials and Methods}

\subsection{Study Population}

As a nationwide representative cross-sectional survey, the KNHANES has been administered to assess the health and nutritional status of Koreans residing in Korea by the Korea Centers for Disease Control and Prevention (KCDC) since 1998, following the National Health Promotion Act. The KNHANES collects data by staged, stratified, clustered, and systematic probability sampling based on sex, age, and geographic area using household registries to represent the entire Korean population living in Korea. To date, the KNHANES has been performed in seven phases including KNHANES phases I (1998), II (2001), III (2005), IV (2007-2009), V (2010-2012), VI (2013-2015), and VII (2016-2018). Among the phases of data, we selected data from 2018 (which is the latest data) to provide timely health statistics results. However, the range of selected data was expanded to 2014 to compensate for the decreased statistical power by exclusion criteria according to the definition of sarcopenia.

To gather information such as health status, health behavior, socioeconomic demographics, laboratory test results, and nutritional status of respondents, the survey consists of three components including a health interview, a health examination, and a nutrition survey among respondents. Health interviews and health examinations are conducted by trained personnel at mobile examination centers, while nutrition surveys are conducted by dietician visits to the homes of participants $[14,15]$.

Individuals aged 60 years or older were included in our analysis from 2014 to 2018 [2,16]. Participants who have any cancers according to the definition of sarcopenia were excluded [16]. We also excluded participants with daily protein intakes under the Recommended Daily Allowance (RDA) for Korean elderly adults $(0.91 \mathrm{~g} / \mathrm{kg} /$ day) to evaluate the proper effects of $\omega 3 \mathrm{FAs}$ on aging-related muscle loss [17]. In the final analysis, a total of 3815 participants (1960 men and 1855 women) were included and analyzed. Approval from an institutional review board (IRB) was not required because the survey did not deal with any sensitive information, only publicly available information. Data from the KNHANES are available for free on the KNHANES website (http://knhanes.cdc.go.kr) for academic research.

\subsection{Definitions of Sarcopenic Obesity, Handgrip Strength, Obesity, and $\omega 3 F A$ Ratio}

Sarcopenic obesity (SO) is a combination of low muscle mass (sarcopenia) and obesity [18]. Sarcopenia is an aging-related loss of muscle mass or decrease in muscular function without other comorbidities such as malignancies $[16,19]$. Because data regarding muscle mass were not available in the 2014-2018 KNHANES, we used decreased muscular function instead of muscle mass loss to diagnose sarcopenia $[16,19,20]$. To assess muscular function, the handgrip strength test was utilized.

Handgrip strength was measured using a digital grip dynamometer (T.K.K.5401; Takei Scientific Instruments Co., Ltd., Niigata, Japan) and defined as the maximally measured grip strength out of three tries with the dominant hand of participants [21]. Differences in handgrip strength among races 
have been reported in previous studies. For instance, the mean handgrip strength of Asian subjects was significantly different from that of Caucasian subjects [22]. Based on this, the Asian Working Group for Sarcopenia (AWGS) recommends using the lower 20th percentile of handgrip strength of each country's health population as the cut-off value to identify low muscle strength for diagnosis of sarcopenia instead of adopting the European-based Europe Working Group on Sarcopenia in Older People 2 (EWGSOP2) cut-off points [16]. To date, there are no standard and nationally representative handgrip strength cut-off values for sarcopenia in Korea, although those based on single-year KNHANES data have been suggested [19]. Therefore, rather than referring to one of the existing values, we determined the cut-off point for sarcopenia in Korean subjects by analyzing the latest five years of KNHANES data in person according to AWGS recommendations to enhance the reliability of our study. The cut-off value of handgrip strength for a sarcopenia diagnosis was the lowest 20th percentile of handgrip strength of people without other comorbidities such as malignancies [16,19], aged from 19 to 80 years, in both sexes (34.5 kg in men and $20.0 \mathrm{~kg}$ in women) [19].

Body mass index (BMI) was calculated as body weight $(\mathrm{kg})$ divided by squared height $\left(\mathrm{m}^{2}\right)$. Obesity based on BMI was defined as equal to or above $25 \mathrm{~kg} / \mathrm{m}^{2}$ [23].

The ratio of daily $\omega 3 \mathrm{FA}$ intake to energy intake was categorized into four quartile groups: Q1, $<0.4$ (both sexes), Q2, 0.4-<0.7 (both sexes), Q3, 0.7-<1.1 (men), 0.7-<1.2 (women), and Q4, $\geq 1.1$ (men), $\geq 1.2$ (women). Hereinafter, we will refer to the ratio of daily $\omega 3$ FA intake to energy intake as the $\omega 3$ FA ratio.

\subsection{Definitions of Other Variables}

Individuals who engaged in moderate physical activity over 150 min per week or who engaged in vigorous physical activity over 75 min per week comprised the group defined as having sufficient physical activity. Men who drank more than seven alcoholic beverages and women who drank more than five alcoholic beverages more than twice a week were categorized as heavy alcohol drinkers [24]. Occupational status was classified into three groups, including (1) manual workers (clerks, service and sales workers, skilled agricultural, forestry, and fishery workers, persons who operate or assemble crafts, equipment, or machines, and elementary workers), (2) office workers (general managers, government administrators, professionals, and simple office workers), and (3) others (unemployed persons, housekeepers, and students). Educational status was divided into four groups as follows: $<6$ years, 6- $<9$ years, $9-<12$ years, and $\geq 12$ years of education. Marital status was categorized into two groups of (1) married and not separated (individuals who were married and living together with their spouse without separation) and (2) single (individuals who were unmarried, separated, divorced, or widowed). Blood pressure (BP) was measured three times in subjects by a standard mercury sphygmomanometer (Baumanometer; Baum Co., Inc., Copiague, NY, USA) and a mean value of the last two measurements was used as the final BP of subjects. Total cholesterol, glucose, and aspartate transaminase (AST) levels were measured by an enzymatic method, hexokinase ultraviolet, and International Federation of Clinical Chemistry (IFCC) techniques without pyridoxal-5-phosphate (P5P), respectively (Hitachi 7600 Automatic Analyzer, Hitachi, Ltd., Tokyo, Japan). High-sensitivity C-reactive protein (hsCRP) values were measured by immunoturbidimetry (Cobas analyzer; F. Hoffmann-La Roche Ltd., Basel, Switzerland).

\subsection{Statistical Analysis}

All data on continuous variables are presented as means \pm standard errors (SEs). Data on categorical variables are presented as percentages \pm SEs. All sampling and weight variables were stratified by sex. Statistical software SAS version 9.4 (SAS Institute Inc., Cary, NC, USA) was used for statistical analysis. This was to account for the intricate sampling design and to provide nationally representative prevalence estimates. Survey regressions and chi-squared $(\chi 2)$ tests were used to compare sexes and quartiles of $\omega 3$ FA ratios. $p$-values were calculated by multiple logistic regression analyses with weighting of the survey design (adjusted with age, body mass index (BMI), 
total cholesterol, systolic BP, fasting plasma glucose, AST, hsCRP, smoking status, alcohol intake, economic status, marital status, education duration, occupation, physical activity, history of diabetes mellitus, history of hypertension, and protein intake). We also estimated adjusted odds ratios (ORs) and $95 \%$ confidence intervals (CIs) by multivariate logistic regression models to investigate factors associated with the sarcopenic obesity group according to the w3FA ratio. All statistical tests were two-tailed, and statistical significance was considered at $p$-values $<0.05$.

\section{Results}

Table 1 presents population characteristics according to sex. The mean ages of men and women were 68.8 and 68.5 years, respectively. Male and female daily energy intakes were 2423.1 and 2013.3 kilocalories (kcal), respectively. Men consumed more macronutrients (carbohydrates, proteins, and fats) than women (all $p$-values $<0.001$ ). However, in terms of the $\omega 3$ FA ratio, the same amount was consumed $(0.9 \%)$ in both sexes $(p$-value $=0.254)$.

Table 1. Population characteristics according to sex.

\begin{tabular}{|c|c|c|c|}
\hline Characteristic & Males & Females & $p$-Value \\
\hline Number & 1960 & 1855 & \\
\hline Age, years & $68.8 \pm 0.2$ & $68.5 \pm 0.2$ & 0.159 \\
\hline BMI, $\mathrm{Kg} / \mathrm{m}^{2}$ & $23.5 \pm 0.1$ & $23.6 \pm 0.1$ & 0.173 \\
\hline Energy intake, Kcal/day & $2423.1 \pm 18.8$ & $2013.3 \pm 17.0$ & $<0.001$ \\
\hline Carbohydrate intake, g/day & $381.6 \pm 3.4$ & $339.0 \pm 3.4$ & $<0.001$ \\
\hline Protein intake, g/day & $88.3 \pm 0.8$ & $73.1 \pm 0.6$ & $<0.001$ \\
\hline Fat intake, g/day & $44.6 \pm 0.8$ & $38.1 \pm 0.6$ & $<0.001$ \\
\hline Daily omega-3/energy intake, \% & $0.9 \pm 0.0$ & $0.9 \pm 0.0$ & 0.254 \\
\hline Total cholesterol, $\mathrm{mg} / \mathrm{dL}$ & $182.4 \pm 1.0$ & $194.6 \pm 1.1$ & $<0.001$ \\
\hline Systolic blood pressure, $\mathrm{mmHg}$ & $125.0 \pm 0.4$ & $127.3 \pm 0.6$ & $<0.001$ \\
\hline Fasting plasma glucose, $\mathrm{mg} / \mathrm{dL}$ & $108.6 \pm 0.7$ & $104.1 \pm 0.7$ & $<0.001$ \\
\hline AST, IU/L & $24.6 \pm 0.3$ & $23.0 \pm 0.2$ & $<0.001$ \\
\hline hsCRP, mg/L & $1.5 \pm 0.1$ & $1.3 \pm 0.1$ & 0.082 \\
\hline Current smoking, \% & $21.7 \pm 1.0$ & $2.4 \pm 0.4$ & $<0.001$ \\
\hline Heavy alcohol intake, \% & $12.9 \pm 0.9$ & $1.8 \pm 0.3$ & $<0.001$ \\
\hline Economic status, $\%$ & & & 0.006 \\
\hline Low & $26.6 \pm 1.2$ & $31.9 \pm 1.3$ & \\
\hline Middle-low & $29.2 \pm 1.2$ & $27.8 \pm 1.3$ & \\
\hline Middle-high & $23.7 \pm 1.0$ & $20.8 \pm 1.1$ & \\
\hline High & $20.5 \pm 1.1$ & $19.6 \pm 1.3$ & \\
\hline Marital status, $\%$ & & & $<0.001$ \\
\hline Married and not separated & $90.9 \pm 0.7$ & $62.2 \pm 1.4$ & \\
\hline Single & $9.1 \pm 0.7$ & $37.8 \pm 1.4$ & \\
\hline Education duration, $\%$ & & & $<0.001$ \\
\hline$<6$ years & $30.8 \pm 1.3$ & $52.9 \pm 1.5$ & \\
\hline $6-<9$ years & $17.8 \pm 1.1$ & $16.8 \pm 1.0$ & \\
\hline $9-<12$ years & $29.2 \pm 1.3$ & $18.6 \pm 1.1$ & \\
\hline$\geq 12$ years & $22.2 \pm 1.2$ & $11.6 \pm 1.0$ & \\
\hline Occupation, \% & & & $<0.001$ \\
\hline Office workers & $11.4 \pm 0.9$ & $4.0 \pm 0.6$ & \\
\hline Manual workers & $40.9 \pm 1.4$ & $30.5 \pm 1.3$ & \\
\hline Other & $47.7 \pm 1.4$ & $65.5 \pm 1.3$ & \\
\hline Sufficient physical activity, \% & $46.5 \pm 1.4$ & $36.8 \pm 1.4$ & $<0.001$ \\
\hline History of diabetes mellitus, $\%$ & $17.0 \pm 1.0$ & $13.0 \pm 0.9$ & 0.002 \\
\hline History of hypertension, \% & $42.1 \pm 1.3$ & $41.5 \pm 1.4$ & 0.782 \\
\hline
\end{tabular}

All data are presented as mean \pm standard errors (SEs) or percentage \pm SEs. Abbreviations: BMI, body mass index; AST, aspartate transaminase; IU/L, international units per liter; hsCRP, high-sensitivity C-reactive protein. 
Table 2 shows participants' characteristics according to $\omega 3$ FA ratio quartile. In men, mean age was not significantly different among the four quartile groups, while daily energy intake and hsCRP levels decreased with increasing quartiles of $\omega 3$ FA ratio. Daily protein intake positively correlated with $\omega 3$ FA ratio quartile. In women, individuals in higher quartiles of $\omega 3$ FA ratio were younger. Daily energy intake decreased with $\omega 3$ FA ratio quartiles, while protein intake increased. Among the four groups, hsCRP levels were not different.

Table 2. Population characteristics according to quartiles of $\omega 3$ FA ratio.

\begin{tabular}{|c|c|c|c|c|c|}
\hline Males & Q1 & Q2 & Q3 & Q4 & $p$-Value \\
\hline & $(<0.4)$ & $(0.4-<0.7)$ & $(0.7-<1.1)$ & $(\geq 1.1)$ & \\
\hline Number & 517 & 483 & 485 & 475 & \\
\hline Age, years & $68.8 \pm 0.3$ & $69.4 \pm 0.3$ & $68.3 \pm 0.3$ & $68.8 \pm 0.3$ & 0.087 \\
\hline BMI, $\mathrm{Kg} / \mathrm{m}^{2}$ & $23.3 \pm 0.1$ & $23.3 \pm 0.1$ & $23.7 \pm 0.1$ & $23.6 \pm 0.1$ & 0.061 \\
\hline Energy intake, Kcal/day & $2495.8 \pm 36.6$ & $2458.4 \pm 36.8$ & $2360.2 \pm 32.6$ & $2378.4 \pm 39.8$ & 0.024 \\
\hline Carbohydrate intake, g/day & $403.0 \pm 7.3$ & $401.7 \pm 6.3$ & $366.2 \pm 5.3$ & $355.7 \pm 5.9$ & $<0.001$ \\
\hline Protein intake, g/day & $82.7 \pm 1.3$ & $86.5 \pm 1.4$ & $88.6 \pm 1.3$ & $95.5 \pm 1.9$ & $<0.001$ \\
\hline Fat intake, g/day & $38.6 \pm 1.3$ & $42.6 \pm 1.4$ & $43.8 \pm 1.3$ & $53.2 \pm 1.7$ & $<0.001$ \\
\hline Daily omega-3/energy intake, \% & $0.3 \pm 0.0$ & $0.5 \pm 0.0$ & $0.9 \pm 0.0$ & $2.0 \pm 0.1$ & $<0.001$ \\
\hline Total cholesterol, $\mathrm{mg} / \mathrm{dL}$ & $185.4 \pm 1.9$ & $181.8 \pm 1.8$ & $183.0 \pm 2.1$ & $179.3 \pm 2.1$ & 0.223 \\
\hline Systolic blood pressure, $\mathrm{mmHg}$ & $126.8 \pm 0.8$ & $126.2 \pm 0.8$ & $123.4 \pm 0.7$ & $123.7 \pm 0.9$ & 0.002 \\
\hline Fasting plasma glucose, $\mathrm{mg} / \mathrm{dL}$ & $111.1 \pm 1.5$ & $106.7 \pm 1.3$ & $109.1 \pm 1.5$ & $107.6 \pm 1.3$ & 0.170 \\
\hline AST, IU/L & $25.7 \pm 0.7$ & $24.2 \pm 0.4$ & $24.3 \pm 0.4$ & $24.2 \pm 0.5$ & 0.283 \\
\hline hsCRP, mg/L & $1.8 \pm 0.2$ & $1.5 \pm 0.2$ & $1.6 \pm 0.1$ & $1.2 \pm 0.1$ & 0.013 \\
\hline Current smoking, \% & $26.3 \pm 2.3$ & $20.0 \pm 2.0$ & $21.5 \pm 2.2$ & $19.2 \pm 2.1$ & 0.113 \\
\hline Heavy alcohol intake, \% & $16.3 \pm 1.8$ & $12.0 \pm 1.6$ & $13.0 \pm 1.8$ & $10.4 \pm 1.6$ & 0.082 \\
\hline Economic status, $\%$ & & & & & 0.037 \\
\hline Low & $32.2 \pm 2.4$ & $26.1 \pm 2.4$ & $22.1 \pm 2.1$ & $25.9 \pm 2.3$ & \\
\hline Middle-low & $29.4 \pm 2.2$ & $29.0 \pm 2.4$ & $28.9 \pm 2.4$ & $29.4 \pm 2.4$ & \\
\hline Middle-high & $19.5 \pm 2.0$ & $22.4 \pm 2.1$ & $27.2 \pm 2.2$ & $25.8 \pm 2.1$ & \\
\hline High & $18.9 \pm 2.1$ & $22.5 \pm 2.4$ & $21.8 \pm 2.2$ & $18.9 \pm 1.9$ & \\
\hline Marital status, $\%$ & & & & & 0.038 \\
\hline Married and not separated & $87.7 \pm 1.6$ & $90.9 \pm 1.5$ & $93.4 \pm 1.2$ & $91.5 \pm 1.5$ & \\
\hline Single & $12.3 \pm 1.6$ & $9.1 \pm 1.5$ & $6.6 \pm 1.2$ & $8.5 \pm 1.5$ & \\
\hline Education duration, $\%$ & & & & & $<0.001$ \\
\hline$<6$ years & $39.7 \pm 2.4$ & $29.5 \pm 2.3$ & $28.7 \pm 2.3$ & $25.1 \pm 2.4$ & \\
\hline $6-<9$ years & $17.8 \pm 1.9$ & $18.1 \pm 2.0$ & $17.3 \pm 2.1$ & $18.0 \pm 2.2$ & \\
\hline $9-<12$ years & $25.2 \pm 2.1$ & $28.4 \pm 2.4$ & $32.3 \pm 2.5$ & $31.1 \pm 2.6$ & \\
\hline$\geq 12$ years & $17.4 \pm 2.1$ & $24.0 \pm 2.3$ & $21.7 \pm 2.1$ & $25.7 \pm 2.2$ & \\
\hline Occupation, \% & & & & & 0.450 \\
\hline Office workers & $10.7 \pm 1.6$ & $10.9 \pm 1.7$ & $12.7 \pm 1.9$ & $11.3 \pm 1.6$ & \\
\hline Manual workers & $46.4 \pm 2.6$ & $41.3 \pm 2.7$ & $38.8 \pm 2.6$ & $37.1 \pm 2.4$ & \\
\hline Other & $42.9 \pm 2.6$ & $47.7 \pm 2.7$ & $48.4 \pm 2.7$ & $51.6 \pm 2.7$ & \\
\hline Sufficient physical activity, $\%$ & $44.7 \pm 2.5$ & $49.0 \pm 2.8$ & $47.3 \pm 2.6$ & $44.8 \pm 2.6$ & 0.633 \\
\hline History of diabetes mellitus, $\%$ & $14.4 \pm 1.7$ & $15.9 \pm 1.9$ & $20.4 \pm 2.2$ & $17.3 \pm 1.8$ & 0.172 \\
\hline History of hypertension, \% & $41.8 \pm 2.4$ & $44.5 \pm 2.6$ & $39.2 \pm 2.5$ & $42.8 \pm 2.7$ & 0.523 \\
\hline
\end{tabular}

All data are presented as mean \pm SEs or percentage \pm SEs. Abbreviations: $\omega 3$ FA, omega- 3 fatty acid; $\omega 3$ FA ratio, ratio of daily omega-3 fatty acid intake to energy intake; BMI, body mass index; AST, aspartate transaminase; hsCRP, high-sensitivity C-reactive protein. 
Table 2. Cont.

\begin{tabular}{|c|c|c|c|c|c|}
\hline Females & Q1 & Q2 & Q3 & Q4 & $p$-Value \\
\hline & $(<0.4)$ & $(0.4-<0.7)$ & $(0.7-<1.2)$ & $(\geq 1.2)$ & \\
\hline Number & 460 & 479 & 454 & 462 & \\
\hline Age, years & $69.5 \pm 0.4$ & $68.9 \pm 0.3$ & $68.0 \pm 0.4$ & $67.6 \pm 0.3$ & $<0.001$ \\
\hline BMI, $\mathrm{Kg} / \mathrm{m}^{2}$ & $23.5 \pm 0.2$ & $23.7 \pm 0.1$ & $23.7 \pm 0.1$ & $23.7 \pm 0.1$ & 0.704 \\
\hline Energy intake, Kcal/day & $2138.0 \pm 40.5$ & $1975.8 \pm 30.0$ & $1945.5 \pm 31.7$ & $1994.2 \pm 34.4$ & 0.002 \\
\hline Carbohydrate intake, g/day & $388.5 \pm 8.7$ & $337.8 \pm 5.8$ & $317.4 \pm 5.6$ & $312.6 \pm 6.7$ & $<0.001$ \\
\hline Protein intake, g/day & $69.5 \pm 1.5$ & $69.4 \pm 1.0$ & $74.8 \pm 1.3$ & $78.7 \pm 1.3$ & $<0.001$ \\
\hline Fat intake, g/day & $30.1 \pm 1.1$ & $34.7 \pm 1.1$ & $40.3 \pm 1.2$ & $47.2 \pm 1.4$ & $<0.001$ \\
\hline Daily omega-3/energy intake, $\%$ & $0.3 \pm 0.0$ & $0.6 \pm 0.0$ & $0.9 \pm 0.0$ & $2.0 \pm 0.1$ & $<0.001$ \\
\hline Total cholesterol, $\mathrm{mg} / \mathrm{dL}$ & $194.9 \pm 2.1$ & $195.1 \pm 2.2$ & $193.9 \pm 2.0$ & $194.4 \pm 2.2$ & 0.979 \\
\hline Systolic blood pressure, $\mathrm{mmHg}$ & $129.2 \pm 1.2$ & $127.1 \pm 0.8$ & $126.1 \pm 0.9$ & $126.9 \pm 1.1$ & 0.211 \\
\hline Fasting plasma glucose, $\mathrm{mg} / \mathrm{dL}$ & $102.9 \pm 1.3$ & $106.4 \pm 1.9$ & $101.6 \pm 1.1$ & $105.5 \pm 1.3$ & 0.062 \\
\hline AST, IU/L & $22.6 \pm 0.4$ & $23.2 \pm 0.7$ & $23.1 \pm 0.4$ & $23.1 \pm 0.4$ & 0.702 \\
\hline hsCRP, mg/L & $1.3 \pm 0.1$ & $1.4 \pm 0.2$ & $1.4 \pm 0.2$ & $1.2 \pm 0.1$ & 0.863 \\
\hline Current smoking, \% & $4.0 \pm 1.2$ & $2.6 \pm 0.9$ & $1.2 \pm 0.5$ & $2.0 \pm 0.9$ & 0.129 \\
\hline Heavy alcohol intake, \% & $2.9 \pm 0.9$ & $1.9 \pm 0.7$ & $1.6 \pm 0.7$ & $0.8 \pm 0.3$ & 0.084 \\
\hline Economic status, $\%$ & & & & & $<0.001$ \\
\hline Low & $36.1 \pm 2.6$ & $36.9 \pm 2.5$ & $24.6 \pm 2.4$ & $29.8 \pm 2.3$ & \\
\hline Middle-low & $32.9 \pm 2.5$ & $25.9 \pm 2.3$ & $26.3 \pm 2.2$ & $26.0 \pm 2.3$ & \\
\hline Middle-high & $17.7 \pm 2.3$ & $18.7 \pm 2.0$ & $26.1 \pm 2.6$ & $20.6 \pm 2.2$ & \\
\hline High & $13.4 \pm 2.1$ & $18.4 \pm 2.4$ & $23.0 \pm 2.2$ & $23.7 \pm 2.5$ & \\
\hline Marital status, $\%$ & & & & & $<0.001$ \\
\hline Married and not separated & $55.3 \pm 2.7$ & $56.7 \pm 2.7$ & $67.6 \pm 2.6$ & $69.2 \pm 2.5$ & \\
\hline Single & $44.7 \pm 2.7$ & $43.3 \pm 2.7$ & $32.4 \pm 2.6$ & $30.8 \pm 2.5$ & \\
\hline Education duration, $\%$ & & & & & $<0.001$ \\
\hline$<6$ years & $64.2 \pm 3.1$ & $55.0 \pm 2.7$ & $45.9 \pm 2.6$ & $47.2 \pm 2.8$ & \\
\hline $6-<9$ years & $15.2 \pm 2.1$ & $16.3 \pm 1.9$ & $17.7 \pm 1.9$ & $17.9 \pm 2.4$ & \\
\hline $9-<12$ years & $13.8 \pm 2.3$ & $18.8 \pm 2.2$ & $22.0 \pm 2.3$ & $19.5 \pm 2.0$ & \\
\hline$\geq 12$ years & $6.8 \pm 1.6$ & $9.8 \pm 1.5$ & $14.3 \pm 1.9$ & $15.3 \pm 1.8$ & \\
\hline Occupation, $\%$ & & & & & 0.782 \\
\hline Office workers & $2.9 \pm 1.1$ & $2.9 \pm 0.9$ & $5.1 \pm 1.2$ & $4.9 \pm 1.0$ & \\
\hline Manual workers & $31.8 \pm 2.5$ & $34.7 \pm 2.3$ & $26.3 \pm 2.3$ & $29.3 \pm 2.2$ & \\
\hline Other & $65.4 \pm 2.5$ & $62.4 \pm 2.4$ & $68.5 \pm 2.4$ & $65.9 \pm 2.3$ & \\
\hline Sufficient physical activity, \% & $34.6 \pm 2.7$ & $35.5 \pm 2.4$ & $39.9 \pm 2.7$ & $36.9 \pm 2.6$ & 0.547 \\
\hline History of diabetes mellitus, $\%$ & $13.0 \pm 1.9$ & $13.5 \pm 1.9$ & $10.5 \pm 1.5$ & $14.9 \pm 1.7$ & 0.327 \\
\hline History of hypertension, \% & $41.1 \pm 2.6$ & $41.3 \pm 2.5$ & $40.1 \pm 2.6$ & $43.6 \pm 2.6$ & 0.804 \\
\hline
\end{tabular}

All data are presented as mean \pm SEs or percentage \pm SEs. Abbreviations: $\omega 3$ FA, omega- 3 fatty acid; $\omega 3$ FA ratio, ratio of daily omega-3 fatty acid intake to energy intake; BMI, body mass index; AST, aspartate transaminase; hsCRP, high-sensitivity C-reactive protein.

Table 3 demonstrates fat intake according to the presence of sarcopenic obesity. Values of $\omega 3 F A$ ratio in women are the only significant difference between groups with sarcopenic obesity and non-sarcopenic obesity. The w3FA ratio in individuals without sarcopenic obesity was significantly higher than in individuals with sarcopenic obesity $(0.8 \%$ in sarcopenic obesity vs. $1.0 \%$ in non-sarcopenic obesity, $p$-value $=0.017$ ). 
Table 3. Ratio of daily total fat and fatty acids intake to energy intake according to the presence of sarcopenic obesity.

\begin{tabular}{cccc}
\hline $\begin{array}{c}\text { Ratio of Daily Total Fat and Fatty } \\
\text { Acids Intake to Energy Intake (\%) }\end{array}$ & Sarcopenic Obesity & Non-Sarcopenic Obesity & $\boldsymbol{p}$-Value \\
\hline Males & & & \\
Total fat & $16.5 \pm 0.6$ & $16.3 \pm 0.2$ & 0.741 \\
SFA & $4.7 \pm 0.2$ & $4.7 \pm 0.1$ & 0.853 \\
MUFA & $5.0 \pm 0.2$ & $5.0 \pm 0.1$ & 0.922 \\
PUFA & $4.9 \pm 0.2$ & $4.6 \pm 0.1$ & 0.177 \\
Omega-3 FA & $1.0 \pm 0.1$ & $0.9 \pm 0.0$ & 0.271 \\
Omega-6 FA & $3.9 \pm 0.2$ & $3.7 \pm 0.1$ & 0.270 \\
Females & $16.8 \pm 0.6$ & $17.1 \pm 0.2$ & 0.633 \\
Total fat & $4.9 \pm 0.2$ & $4.9 \pm 0.1$ & 0.879 \\
SFA & $5.2 \pm 0.2$ & $5.2 \pm 0.1$ & 0.995 \\
MUFA & $4.6 \pm 0.2$ & $5.0 \pm 0.1$ & 0.121 \\
PUFA & $0.8 \pm 0.0$ & $1.0 \pm 0.0$ & 0.017 \\
Omega-3 FA & $3.8 \pm 0.2$ & $4.0 \pm 0.1$ & 0.334 \\
Omega-6 FA & Abbreviations: SFA saturated fatty acids; MUFA
\end{tabular}

All data are presented as mean ( \pm standard errors). Abbreviations: SFA, saturated fatty acids; MUFA, monounsaturated fatty acids; PUFA, polyunsaturated fatty acids; FA, fatty acids.

Figure 1 presents the prevalence of sarcopenic obesity according to quartile of $\omega 3$ FA ratio. There was no significant difference in sarcopenic obesity prevalence among male quartile groups, while the female prevalence was marginally different $(p$-value $=0.055)$. The lowest quartile of $\omega 3$ FA ratio had the highest prevalence $(17.4 \%)$ of sarcopenic obesity in women, while the highest quartile of $\omega 3$ FA ratio showed the lowest $(10.1 \%)$.

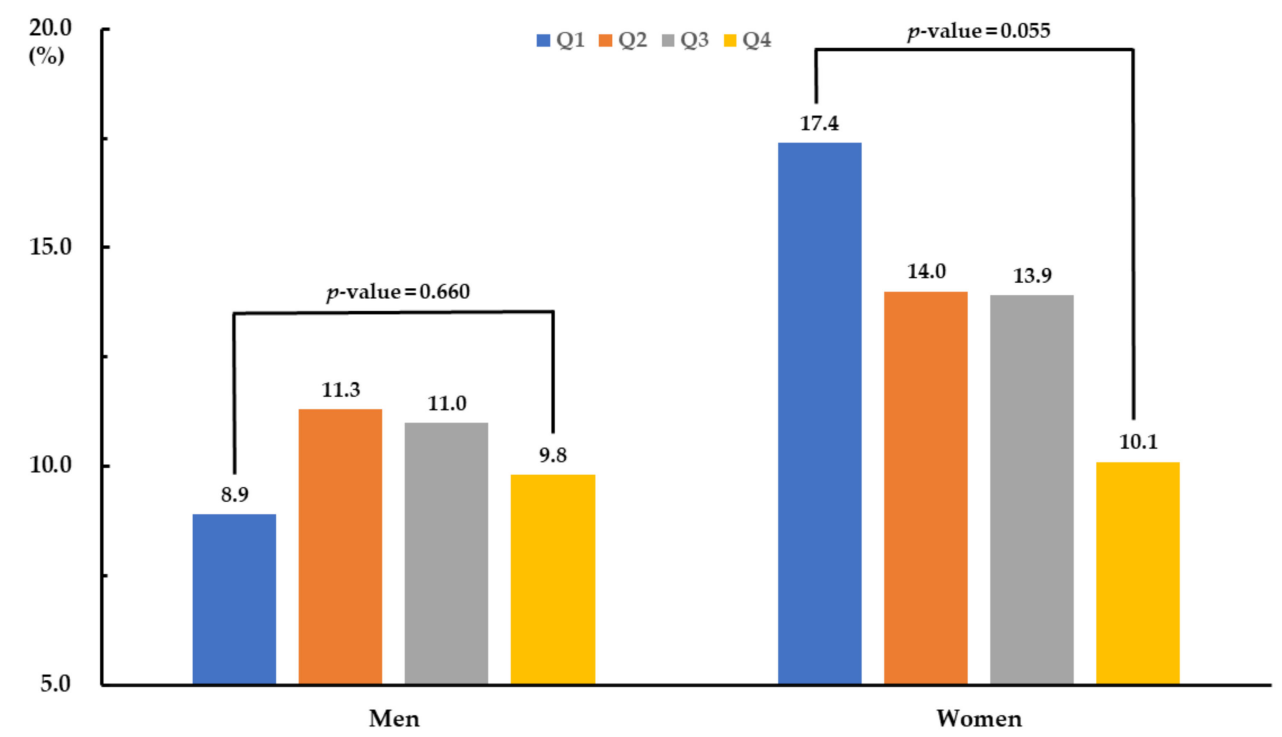

Figure 1. Prevalence of sarcopenic obesity according to quartile group of omega-3 fatty acid ratio.

Table 4 demonstrates logistic regression models according to $\omega 3$ FA ratio quartile. Compared with Q1, ORs (95\% CIs) for sarcopenic obesity of Q2, Q3, and Q4 of w3FA ratio were 1.288 (0.776-2.138), 1.298 (0.804-2.097), and 1.118 (0.701-1.782) respectively, in men and 0.807 (0.534-1.221), 0.847 (0.552-1.299), and $0.603(0.376-0.967)$ respectively, in women after adjusting for age. After full adjustment, ORs (95\% CIs) of Q2, Q3, and Q4 of $\omega 3$ FA ratio were 1.563 (0.802-3.047), 1.246 (0.611-2.542), and 0.924 (0.458-1.864) respectively, in men, and 0.663 (0.379-1.160), $0.640(0.372-1.102)$, and 0.246 (0.113-0.534) respectively, in women. 
Table 4. Adjusted odds ratios (ORs) and 95\% confidence intervals (CIs) of sarcopenic obesity by quartiles of $\omega 3$ FA ratio.

\begin{tabular}{ccccc}
\hline Males & Q1 & Q2 & Q3 & Q4 \\
& $(<0.4)$ & $(0.4-<0.7)$ & $(0.7-<1.1)$ & $(\geq 1.1)$ \\
\hline Model 1 & 1 (ref) & $1.288(0.776-2.138)$ & $1.298(0.804-2.097)$ & $1.118(0.701-1.782)$ \\
Model 2 & 1 (ref) & $1.668(0.868-3.205)$ & $1.160(0.592-2.272)$ & $1.000(0.512-1.953)$ \\
Model 3 & 1 (ref) & $1.563(0.802-3.047)$ & $1.246(0.611-2.542)$ & $0.924(0.458-1.864)$ \\
\hline Females & Q1 & Q2 & Q3 & Q4 \\
\hline & $(<0.4)$ & $(0.4-<0.7)$ & $(0.7-<1.2)$ & $(\geq 1.2)$ \\
\hline Model 1 & 1 (ref) & $0.807(0.534-1.221)$ & $0.847(0.552-1.299)$ & $0.603(0.376-0.967)$ \\
Model 2 & 1 (ref) & $0.604(0.355-1.028)$ & $0.649(0.394-1.072)$ & $0.282(0.129-0.617)$ \\
\hline Model 3 & 1 (ref) & $0.663(0.379-1.160)$ & $0.640(0.372-1.102)$ & $0.246(0.113-0.534)$ \\
\hline
\end{tabular}

Odds ratios (ORs) and 95\% confidence intervals (CIs) were calculated using weighted multivariate logistic regression analyses. Model 1: Adjusted for age. Model 2: Adjusted for age, BMI, total cholesterol, systolic blood pressure, fasting plasma glucose, AST, and hsCRP. Model 3: Adjusted for current smoking status, heavy alcohol intake, economic status, marital status, education duration, occupation, sufficient physical activity, history of diabetes mellitus, history of hypertension, and protein intake in addition to variables of Model 2. Abbreviation: w3FA ratio, ratio of daily w3FA intake to energy intake; AST, aspartate transaminase; hsCRP, high-sensitivity C-reactive protein.

\section{Discussion}

This study finds that the $\omega 3$ FA ratio was significantly higher in women without $\mathrm{SO}$ than in women with SO. Logistic regression models demonstrate that the $\omega 3$ FA ratio was inversely associated with the prevalence of SO in elderly females (but not in males), even after adjusting for confounding variables.

To date, resistance exercise and protein supplementation have been generally accepted as the most effective lifestyle changes to prevent sarcopenia $[25,26]$. Thus, the two are recommended for the prevention and management of sarcopenia [27,28]. However, resistance exercise and protein supplementation have not been shown to consistently lead to significantly positive results in all the research [28]. Anabolic resistance, which is a decreased response to anabolic stimuli such as physical exercise and ingested protein, arises in the protein metabolism of human bodies as people get older [29]. Thus, we have hypothesized that an additional approach for overcoming anabolic resistance would be necessary along with exercise and protein intake to prevent and treat sarcopenia in the elderly.

In the past, many researchers hypothesized that anti-inflammatory effects of $\omega 3$ FAs were mainly related to observed prevention effects in sarcopenia such as cachexia in cancers or chronic diseases [30]. Today, overcoming age-related anabolic resistance is considered to be the main mechanism of w3FAs in the prevention of sarcopenia [31]. On the molecular level, the mTORp/70s6k signaling pathway is considered an integral point of age-related anabolic resistance [6]. The mammalian target of rapamycin (mTOR) is a sensing effector of hormone and nutrient availability that controls two key translation initiation promoters, $\mathrm{S} 6$ kinase (S6K) and $4 \mathrm{E}$ binding protein (BP), of eukaryotic initiation factor eIF4E [5]. $\omega 3$ FAs make this effector more sensitive to anabolic stimuli, and thus, promote cellular mRNA translation of proteins [5,31]. Prostaglandin I2 (prostacyclin, PGI2) triggers cyclic adenosine monophosphate (cAMP) production through prostacyclin receptors at the surface of preadipocytes. cAMP activates the protein kinase A pathway that promotes the differentiation of preadipocytes into adipocytes. $\omega 3$ FAs inhibit the synthesis of PGI2 at the level of cyclooxygenases (COX) by competing with omega- 6 fatty acids (w6FAs), which are precursors of PGI2, and consequently inhibit lipogenesis [32,33].

$\omega 3 F A s$ are also found to increase the activity of peroxisomal and mitochondrial enzymes for $\beta$-oxidation of fatty acids. $\omega 3$ FAs mainly induce gene expression of acyl-CoA oxidase, which is the key enzyme of fatty acids $\beta$-oxidation in peroxisomes [10]. Concurrently, w3FAs elevate the activity of carnitine palmitoyltransferase II, which is a shuttle of fatty acids into mitochondria for further $\beta$-oxidation. Therefore, $\omega 3$ FAs accelerate overall lipid oxidation in both peroxisomes and mitochondria [34]. 
As mentioned above, previous studies show that $\omega 3$ FAs have the potential to prevent and treat SO. However, because most studies have explored the effects of $\omega 3$ FAs either for sarcopenia [6] or obesity (but not for both [35]), previous studies alone cannot directly evaluate the beneficial effects of $\omega 3 \mathrm{FAs}$ for SO. By directly investigating the association between $\omega 3 \mathrm{FA}$ intake and the prevalence of $\mathrm{SO}$, this study differentiates its clinical significance from previous studies to date.

A noteworthy point of our findings is that there is a significant association between $\omega 3$ FA ratio and prevalence of $\mathrm{SO}$ in older women but not in men. Although the exact mechanism remains unknown, women might have more capacity for improvement with w3FAs compared to men [36]. Unlike men, the muscle strength of women does not usually increase to its optimal level in response to resistance exercise; thereby, most women get greater muscle function reservoir to enhance with the intake of $\omega 3 F A s[37]$. The innate biologically sexual differences in the pattern of muscle mass and strength changes with age may also explain these differences. Peak muscle mass and strength of women are lower than in men [38]. Furthermore, the muscle decline in women occurs earlier due to menopause and is steeper than in men, particularly after menopause [39]. Menopause is a major risk factor for sarcopenia and SO [40]. In the present study, only elderly individuals were included, indicating the possibility that most of the women were postmenopausal. Considering the differences in changes between men and women in the pattern of muscle mass and strength with aging, supplementation of muscle-preserving nutrients, including $\omega 3 \mathrm{FA}$, may show a greater preventive effect against $\mathrm{SO}$ in women than in men.

Comparing the intake of $\omega 3$ FA to that in Western countries such as the US and European countries, Koreans are known to consume more $\omega 3 \mathrm{FA}$. We can compare this more reliably by measuring the blood level of eicosapentaenoic acid (EPA) and docosahexaenoic acid (DHA) than by the $24 \mathrm{~h}$ recall method because we can exclude the potential for reporting or recall bias [40,41]. The blood EPA and DHA levels in Koreans are more than twice those of US subjects and higher than most of those in European countries, except Scandinavia [42].

Several limitations should be considered in interpreting this study. First, we defined sarcopenia not directly by measuring muscle mass using body composition examination such as dual-energy X-ray absorptiometry, but indirectly, by measuring muscle function using a handgrip strength test. Because handgrip strength is a reliable predictor of clinical outcomes of low muscle mass, handgrip strength can be used as an indicator to diagnose sarcopenia $[16,19]$. Second, it is difficult to conclude causality from this study because it was cross-sectionally designed without any follow-up. Third, because we use the data of dietary w3FA intake gathered by a $24 \mathrm{~h}$ recall method instead of actual measurements of $\omega 3$ FAs in the red blood cell membrane or plasma of subjects, we cannot completely exclude the potential for reporting or recall bias from this study.

In spite of these limitations, this study has several strengths that distinguish our research from previous studies. First, this study is derived from a population-based sample from a complex survey design, seeking to produce estimates that accurately represent the Korean population. Second, to the best of our knowledge, this is the first Korean study to evaluate the association of $\omega 3$ FA intake and $\omega 3 \mathrm{FA}$ ratio with the prevalence of $\mathrm{SO}$ using nationally representative data. Third, to minimize confounding effects of several factors, especially protein levels, we analyzed the data not only by adjusting for variables such as daily protein intake but also by using the w3FA ratio instead of measured amounts of $\omega 3$ FAs. Fourth, the definition of sarcopenia was not quantitatively measured by muscle mass but instead was functionally evaluated using handgrip strength. To predict clinical outcomes in elderly people, muscular functionality such as handgrip strength is more important than the quantity of muscle mass [20,43].

\section{Conclusions}

In conclusion, this study shows that an increased $\omega 3$ FA ratio-based intake is associated with a decreased prevalence of $\mathrm{SO}$ in elderly females. This means that interventions to increase dietary $\omega 3 \mathrm{FA}$ ratio may help with the prevention and management of SO in elderly females. However, longitudinally 
designed cohort studies or interventional studies are further needed to confirm causality between the $\omega 3 \mathrm{FA}$ ratio and the prevalence of $\mathrm{SO}$ and to determine whether a diet with an increased $\omega 3 \mathrm{FA}$ ratio might be an effective strategy to prevent and manage $\mathrm{SO}$ in specific populations.

Author Contributions: Conceptualization, H.-T.K., J.-w.L., Y.K. and W.Y.; methodology, H.-T.K., J.-w.L., and W.Y.; statistical analysis, J.-w.L. and W.Y.; interpretation, H.-T.K., J.-w.L., Y.K., J.H.L., and W.Y.; writing-original draft preparation, H.-T.K., J.-w.L., Y.K., J.H.L., and W.Y.; funding acquisition, Y.K. All authors have read and agreed to the published version of the manuscript.

Funding: This work was supported by Korea Institute of Planning and Evaluation for Technology in Food, Agriculture and Forestry (IPET) through its future innovative food technology development program, funded by Ministry of Agriculture, Food and Rural Affairs (MAFRA) (grant number: IPET319045032HD060).

Conflicts of Interest: The authors declare no conflict of interest.

\section{References}

1. Saini, A.; Sharples, A.P.; Al-Shanti, N.; Stewart, C.E. Omega-3 fatty acid EPA improves regenerative capacity of mouse skeletal muscle cells exposed to saturated fat and inflammation. Biogerontology 2017, 18, 109-129. [CrossRef] [PubMed]

2. Kim, T.N.; Yang, S.J.; Yoo, H.J.; Lim, K.I.; Kang, H.J.; Song, W.; Seo, J.A.; Kim, S.G.; Kim, N.H.; Baik, S.H.; et al. Prevalence of sarcopenia and sarcopenic obesity in Korean adults: The Korean Sarcopenic Obesity Study (KSOS). Nat. Précéd. 2009, 33, 885-892. [CrossRef]

3. Riechman, S.E.; Schoen, R.E.; Weissfeld, J.L.; Thaete, F.L.; Hamman, R.F. Association of Physical Activity and Visceral Adipose Tissue in Older Women and Men. Obes. Res. 2002, 10, 1065-1073. [CrossRef]

4. Wannamethee, S.G.; Atkins, J.L. Muscle loss and obesity: The health implications of sarcopenia and sarcopenic obesity. In Proceedings of the Nutrition Society; Cambridge University Press (CUP): Cambridge, UK, 2015; Volume 74, pp. 405-412.

5. Gingras, A.-A.; White, P.J.; Chouinard, P.Y.; Julien, P.; Davis, T.A.; Dombrowski, L.; Couture, Y.; Dubreuil, P.; Myre, A.; Bergeron, K.; et al. Long-chain omega-3 fatty acids regulate bovine whole-body protein metabolism by promoting muscle insulin signalling to the Akt-mTOR-S6K1 pathway and insulin sensitivity. J. Physiol. 2007, 579, 269-284. [CrossRef] [PubMed]

6. Smith, G.I.; Atherton, P.; Reeds, D.N.; Mohammed, B.S.; Rankin, D.; Rennie, M.J.; Mittendorfer, B. Dietary omega-3 fatty acid supplementation increases the rate of muscle protein synthesis in older adults: A randomized controlled trial. Am. J. Clin. Nutr. 2011, 93, 402-412. [CrossRef] [PubMed]

7. Pisani, D.F.; Amri, E.-Z.; Ailhaud, G. Disequilibrium of polyunsaturated fatty acids status and its dual effect in modulating adipose tissue development and functions. OCL 2015, 22, D405. [CrossRef]

8. Simopoulos, A.P. An Increase in the Omega-6/Omega-3 Fatty Acid Ratio Increases the Risk for Obesity. Nutrients 2016, 8, 128. [CrossRef]

9. Baillie, R.; Takada, R.; Nakamura, M.; Clarke, S. Coordinate induction of peroxisomal acyl-CoA oxidase and UCP-3 by dietary fish oil: A mechanism for decreased body fat deposition. Prostaglandins Leukot. Essent. Fat. Acids 1999, 60, 351-356. [CrossRef]

10. Ukropec, J.; Reseland, J.E.; Gašperíková, D.; Demcáková, E.; Madsen, L.; Berge, R.K.; Rustan, A.C.; Klimes, I.; Drevon, C.A.; Sebokova, E. The hypotriglyceridemic effect of dietary n-3 FA is associated with increased $\beta$-oxidation and reduced leptin expression. Lipids 2003, 38, 1023-1029. [CrossRef]

11. Wolfe, R.R.; Miller, S.L. The Recommended Dietary Allowance of Protein: A misunderstood concept. JAMA 2008, 299, 2891-2893. [CrossRef]

12. Pepersack, T.; Corretge, M.; Beyer, I.; Namias, B.; Andre, S.; Benoit, F.; Mergam, A.; Simonetti, C. Examining the effect of intervention to nutritional problems of hospitalized elderly: A pilot project. J. Nutr. Health Aging 2002, 6, 306-310. [PubMed]

13. Houston, D.K.; Nicklas, B.J.; Ding, J.; Harris, T.B.; Tylavsky, F.A.; Newman, A.B.; Lee, J.S.; Sahyoun, N.R.; Visser, M.; Kritchevsky, S.B.; et al. Dietary protein intake is associated with lean mass change in older, community-dwelling adults: The Health, Aging, and Body Composition (Health ABC) Study. Am. J. Clin. Nutr. 2008, 87, 150-155. [CrossRef] [PubMed]

14. Kim, Y. The Korea National Health and Nutrition Examination Survey (KNHANES): Current Status and Challenges. Epidemiol. Health 2014, 36, e2014002. [CrossRef] 
15. Kweon, S.; Kim, Y.; Jang, M.-J.; Kim, Y.; Kim, K.; Choi, S.; Chun, C.; Khang, Y.-H.; Oh, K. Data resource profile: The Korea National Health and Nutrition Examination Survey (KNHANES). Int. J. Epidemiol. 2014, 43, 69-77. [CrossRef] [PubMed]

16. Chen, L.-K.; Woo, J.; Assantachai, P.; Auyeung, T.-W.; Chou, M.-Y.; Iijima, K.; Jang, H.C.; Kang, L.; Kim, M.; Kim, S.; et al. Asian Working Group for Sarcopenia: 2019 Consensus Update on Sarcopenia Diagnosis and Treatment. J. Am. Med. Dir. Assoc. 2020, 21, 300-307.e2. [CrossRef] [PubMed]

17. Kwon, D.H.; Park, H.A.; Cho, Y.-G.; Kim, K.; Kim, N.H. Different Associations of Socioeconomic Status on Protein Intake in the Korean Elderly Population: A Cross-Sectional Analysis of the Korea National Health and Nutrition Examination Survey. Nutrients 2019, 12, 10. [CrossRef]

18. Lee, D.-C.; Shook, R.P.; Drenowatz, C.; Blair, S.N. Physical activity and sarcopenic obesity: Definition, assessment, prevalence and mechanism. Futur. Sci. OA 2016, 2, FSO127. [CrossRef] [PubMed]

19. Yoo, J.-I.; Choi, H.; Ha, Y.-C. Mean Hand Grip Strength and Cut-off Value for Sarcopenia in Korean Adults Using KNHANES VI. J. Korean Med. Sci. 2017, 32, 868-872. [CrossRef]

20. Cruz-Jentoft, A.J.; Baeyens, J.P.; Bauer, J.M.; Boirie, Y.; Cederholm, T.; Landi, F.; Martin, F.C.; Michel, J.-P.; Rolland, Y.; Schneider, S.M.; et al. Sarcopenia: European consensus on definition and diagnosis: Report of the European Working Group on Sarcopenia in Older People. Age Ageing 2010, 39, 412-423. [CrossRef]

21. Roberts, H.C.; Denison, H.J.; Martin, H.J.; Patel, H.P.; Syddall, H.; Cooper, C.; Sayer, A.A. A review of the measurement of grip strength in clinical and epidemiological studies: Towards a standardised approach. Age Ageing 2011, 40, 423-429. [CrossRef]

22. Chen, L.-K.; Liu, L.-K.; Woo, J.; Assantachai, P.; Auyeung, T.-W.; Bahyah, K.S.; Chou, M.-Y.; Hsu, P.-S.; Krairit, O.; Lee, J.S.; et al. Sarcopenia in Asia: Consensus Report of the Asian Working Group for Sarcopenia. J. Am. Med. Dir. Assoc. 2014, 15, 95-101. [CrossRef] [PubMed]

23. Paik, D.W.; Han, K.; Kang, S.W.; Ham, D.-I.; Kim, S.J.; Chung, T.-Y.; Lim, D.H. Differential effect of obesity on the incidence of retinal vein occlusion with and without diabetes: A Korean nationwide cohort study. Sci. Rep. 2020, 10, 1-9. [CrossRef] [PubMed]

24. World Health Organization. International Guide for Monitoring Alcohol Consumption and Related Harm; World Health Organization: Geneva, Switzerland, 2000.

25. Cruz-Jentoft, A.J.; Landi, F.; Schneider, S.M.; Zúñiga, C.; Arai, H.; Boirie, Y.; Chen, L.-K.; Fielding, R.A.; Martin, F.C.; Michel, J.-P.; et al. Prevalence of and interventions for sarcopenia in ageing adults: A systematic review. Report of the International Sarcopenia Initiative (EWGSOP and IWGS). Age Ageing 2014, 43, 748-759. [CrossRef] [PubMed]

26. Beaudart, C.; Rabenda, V.; Simmons, M.; Geerinck, A.; De Carvalho, I.A.; Reginster, J.-Y.; Thiyagarajan, J.A.; Bruyère, O. Effects of Protein, Essential Amino Acids, B-Hydroxy B-Methylbutyrate, Creatine, Dehydroepiandrosterone and Fatty Acid Supplementation on Muscle Mass, Muscle Strength and Physical Performance in Older People Aged 60 Years and Over. A Systematic Review of the Literature. J. Nutr. Health Aging 2018, 22, 117-130. [CrossRef] [PubMed]

27. Bauer, J.; Biolo, G.; Cederholm, T.; Cesari, M.; Cruz-Jentoft, A.J.; Morley, J.E.; Phillips, S.; Sieber, C.; Stehle, P.; Teta, D.; et al. Evidence-Based Recommendations for Optimal Dietary Protein Intake in Older People: A Position Paper From the PROT-AGE Study Group. J. Am. Med. Dir. Assoc. 2013, 14, 542-559. [CrossRef]

28. Beaudart, C.; Dawson, A.; Shaw, S.C.; Harvey, N.C.; Kanis, J.A.; Binkley, N.; Reginster, J.Y.; Chapurlat, R.; Chan, D.C.; Bruyère, O.; et al. Nutrition and physical activity in the prevention and treatment of sarcopenia: Systematic review. Osteoporos. Int. 2017, 28, 1817-1833. [CrossRef]

29. Dupont, J.; Dedeyne, L.; Dalle, S.; Koppo, K.; Gielen, E.E. The role of omega-3 in the prevention and treatment of sarcopenia. Aging Clin. Exp. Res. 2019, 31, 825-836. [CrossRef]

30. Yan, Y.; Jiang, W.; Spinetti, T.; Tardivel, A.; Castillo, R.; Bourquin, C.; Guarda, G.; Tian, Z.; Tschopp, J.; Zhou, R. Omega-3 Fatty Acids Prevent Inflammation and Metabolic Disorder through Inhibition of NLRP3 Inflammasome Activation. Immunity 2013, 38, 1154-1163. [CrossRef]

31. Di Girolamo, F.G.; Situlin, R.; Mazzucco, S.; Valentini, R.; Toigo, G.; Biolo, G. Omega-3 fatty acids and protein metabolism: Enhancement of anabolic interventions for sarcopenia. Curr. Opin. Clin. Nutr. Metab. Care 2014, 17, 145-150. [CrossRef]

32. Aubert, J.; Saint-Marc, P.; Belmonte, N.; Dani, C.; Negrel, R.; Ailhaud, G. Prostacyclin IP receptor up-regulates the early expression of C/EBP $\beta$ and C/EBP $\delta$ in preadipose cells. Mol. Cell. Endocrinol. 2000, 160, 149-156. [CrossRef] 
33. Vassaux, G.; Gaillard, D.; Ailhaud, G.; Negrel, R. Prostacyclin is a specific effector of adipose cell differentiation. Its dual role as a cAMP- and Ca(2+)-elevating agent. J. Biol. Chem. 1992, 267, 11092-11097. [PubMed]

34. Ide, T.; Kobayashi, H.; Ashakumary, L.; Rouyer, I.A.; Takahashi, Y.; Aoyama, T.; Hashimoto, T.; Mizugaki, M. Comparative effects of perilla and fish oils on the activity and gene expression of fatty acid oxidation enzymes in rat liver. Biochim. Biophys. Acta BBA Mol. Cell Biol. Lipids 2000, 1485, 23-35. [CrossRef]

35. Couet, C.; Delarue, J.; Ritz, P.; Antoine, J.-M.; Lamisse, F. Effect of dietary fish oil on body fat mass and basal fat oxidation in healthy adults. Int. J. Obes. 1997, 21, 637-643. [CrossRef] [PubMed]

36. Da Boit, M.; Sibson, R.; Sivasubramaniam, S.; Meakin, J.R.; Greig, C.A.; Aspden, R.M.; Thies, F.; Jeromson, S.; Hamilton, D.L.; Speakman, J.R.; et al. Sex differences in the effect of fish-oil supplementation on the adaptive response to resistance exercise training in older people: A randomized controlled trial. Am. J. Clin. Nutr. 2017, 105, 151-158. [CrossRef] [PubMed]

37. Da Boit, M.; Sibson, R.; Meakin, J.R.; Aspden, R.M.; Thies, F.; Mangoni, A.A.; Gray, S.R. Sex differences in the response to resistance exercise training in older people. Physiol. Rep. 2016, 4, e12834. [CrossRef] [PubMed]

38. Neder, J.A.; Nery, L.E.; Andreoni, S.; Whipp, B.J. Maximal aerobic power and leg muscle mass and strength related to age in non-athletic males and females. Graefe's Arch. Clin. Exp. Ophthalmol. 1999, 79, 522-530. [CrossRef]

39. Greeves, J.P.; Cable, N.T.; Reilly, T.; Kingsland, C. Changes in muscle strength in women following the menopause: A longitudinal assessment of the efficacy of hormone replacement therapy. Clin. Sci. 1999, 97, 79-84. [CrossRef]

40. Fratesi, J.A.; Hogg, R.C.; Young-Newton, G.S.; Patterson, A.C.; Charkhzarin, P.; Thomas, K.B.; Sharratt, M.T.; Stark, K.D. Direct quantitation of omega-3 fatty acid intake of Canadian residents of a long-term care facility. Appl. Physiol. Nutr. Metab. 2009, 34, 1-9. [CrossRef]

41. Patterson, A.C.; Metherel, A.H.; Hanning, R.M.; Stark, K.D. The percentage of DHA in erythrocytes can detect non-adherence to advice to increase EPA and DHA intakes. Br. J. Nutr. 2013, 111, 270-278. [CrossRef]

42. Stark, K.D.; Van Elswyk, M.E.; Higgins, M.R.; Weatherford, C.A.; Salem, J.N. Global survey of the omega-3 fatty acids, docosahexaenoic acid and eicosapentaenoic acid in the blood stream of healthy adults. Prog. Lipid Res. 2016, 63, 132-152. [CrossRef]

43. Sinclair, M.; Chapman, B.; Hoermann, R.; Angus, P.W.; Testro, A.; Scodellaro, T.; Gow, P.J. Handgrip Strength Adds More Prognostic Value to the Model for End-Stage Liver Disease Score Than Imaging-Based Measures of Muscle Mass in Men with Cirrhosis. Liver Transplant. 2019, 25, 1480-1487. [CrossRef] [PubMed]

Publisher's Note: MDPI stays neutral with regard to jurisdictional claims in published maps and institutional affiliations.

(C) 2020 by the authors. Licensee MDPI, Basel, Switzerland. This article is an open access article distributed under the terms and conditions of the Creative Commons Attribution (CC BY) license (http://creativecommons.org/licenses/by/4.0/). 